\title{
Ferramenta de Apoio ao Ensino de Estimativa de Software com Planning Poker
}

\author{
André Augusto Tissot ${ }^{1}$, Maria Cláudia Figueiredo Pereira Emer ${ }^{1}$, Laudelino \\ Cordeiro Bastos ${ }^{1}$ \\ ${ }^{1}$ Programa de Pós-Graduação em Computação Aplicada (PPGCA) \\ Departamento de Informática (DAINF) \\ Universidade Tecnológica Federal do Paraná (UTFPR), Curitiba, Brasil \\ andre_a_tissoteyahoo.com.br, mclaudia@dainf.ct.utfpr.edu.br, \\ laudelinobastos@uol.com.br
}

\begin{abstract}
This study describes the development and utilization of a web application to support the teaching of Software Estimation with Planning Poker and the data analysis of the impact of revising previous similar software estimates when conducting software estimates in a Planning Poker context. The behavior of 14 teams that made estimates was analyzed. Among these teams, 11 of them had an improvement in accuracy. In only 3 of them, estimates had a decreased in accuracy. The use of the software tool improved estimation of effort in most of the cases.
\end{abstract}

Resumo. Este trabalho descreve o desenvolvimento e uso de uma ferramenta de apoio ao ensino de Estimativa de Software com Planning Poker e, também, a análise dos dados coletados para a investigação do impacto da revisão de dados históricos de esforço de software na estimativa do esforço utilizando Planning Poker. Foi analisado o comportamento de 14 grupos de estimativas. Dentre esses grupos, 11 grupos tiveram uma melhora da acurácia das estimativas e 3 grupos tiveram uma piora da acurácia das estimativas. Dessa forma, o uso da ferramenta de software melhorou a estimativa de esforço na maioria dos casos analisados.

\section{Introdução}

A estimativa de esforço de desenvolvimento de software é o processo de prever o esforço necessário para desenvolver um software. Subestimar o esforço para o desenvolvimento de softwares pode levar à entrega dos mesmos com uma qualidade menor, além de causar impacto na reputação e na competitividade das organizações de desenvolvimento de software. Porém, superestimar o custo pode resultar em perda de oportunidades interessantes de negócio, ou seja, uma organização pode deixar de desenvolver um novo software por considerar não ter tempo hábil ou pessoas disponíveis para isto. Desta forma, é importante treinar alunos para fazerem estimativas acuradas sobre projetos reais.

Os projetos de software, em média, atingem $30 \%$ de precisão em suas estimativas de software [Jørgensen e Grimstad 2008], o que sugere que o planejamento, como é conduzido hoje, não se mostra confiável o suficiente e, desta forma, o mesmo pode levar ao fracasso dos projetos. 
O método mais comum na indústria de software para realização de estimativas é o baseado em julgamento de especialista, no qual o responsável pela estimativa tem de conhecer profundamente o objetivo da atividade e da eficiência dos profissionais que irão executá-la, e sendo em média tão preciso quanto o uso de modelos formais de estimativa [Jørgensen 2013]. No entanto, a habilidade humana de refletir sobre suas estimativas e melhorá-las é surpreendentemente pobre [Jørgensen e Gruschke 2009]. Verificando a literatura, não foram encontradas referências sobre a influência da revisão de atividades executadas antes da realização de estimativas baseadas em julgamento do especialista.

O Planning Poker é um método de estimativa baseado em julgamento do especialista introduzido por Grenning [2002] e popularizado por Cohn [2005]. As informações geradas pela discussão no Planning Poker [Grenning 2002] não são guardadas pelos participantes, nem quando realizadas por equipes locais, pela informalidade aparente do método, nem quando realizadas por times geograficamente distribuídos, pela falta de suporte das ferramentas de mercado especializadas em Planning Poker. E como esse conhecimento se perde, não há como aproveitá-lo em estimativas futuras.

Este trabalho teve como objetivo apresentar o desenvolvimento e a análise de uma Ferramenta de Apoio ao Ensino de Estimativa de Software com Planning Poker, por meio de experimentos com alunos, com e sem revisão de estimativas similares anteriores.

Este documento está estruturado da seguinte forma: a Seção 2 apresenta o Planning Poker, a Seção 3 apresenta os trabalhos relacionados, a Seção 4 descreve a metodologia utilizada para desenvolver o projeto, a Seção 5 descreve os resultados, as análises e as discussões e a Seção 6 apresenta as conclusões obtidas durante a realização dos experimentos.

\section{Planning Poker}

A estimativa de esforço de software é uma etapa importante da análise de viabilidade de projetos e sua acurácia ainda carece de melhoria, pois como exibido por Gruschke e Jørgensen [2008] em seus experimentos, estimativas baseadas em Julgamento do Especialista tiveram diferença entre esforço estimado mediano e esforço real entre $8 \%$ e $63 \%$, o que aponta a instabilidade da técnica.

Planning Poker é um método de estimativa baseado em julgamento do especialista amplamente utilizado em métodos ágeis, especialmente Scrum e Extreme Programming [Mahnič e Hovelja 2012].

Segundo [Raith et al. 2013], o fluxo de execução do Planning Poker, envolve todos os membros do time de desenvolvimento, como: estimadores, um moderador (um Scrum Master, por exemplo) e, opcionalmente, um representante do cliente.

Comparado também com a média das estimativas, o processo necessário para realização do Planning Poker oferece estimativas mais acuradas e menos otimistas. Além do fato de estimativas realizadas em grupo resultarem em estimativas mais acuradas que estimativas individuais [Raith et al. 2013]. 
Embora o método de estimativa Planning Poker se baseie em estimativas humanas e não utilize análises como medidas históricas ou analogia, uma abordagem para incrementar a sua eficiência é utilizar um repositório histórico [Desharnais et al. 2011].

\section{Trabalhos Relacionados}

Ambientes computacionais para apoio ao ensino, relacionados a requisitos, tem sido desenvolvidos com diferentes vertentes. Santos et al. (2013) desenvolveram um jogo para ensino de conceitos de requisitos em sistemas ubíquos. Zuppiroli, Ciancarini e Gabbrielli (2012) desenvolveram um RPG (role-playing game ou jogo de interpretação de papéis) que permite que requisitos possam ser discutidos e avaliados em um laboratório de engenharia de software. Thiry, Zoucas e Gonçalves (2010) criaram um jogo educativo, que faz uso de aspectos lúdicos e de desafios para promover a aprendizagem da Engenharia de Requisitos. Vargas et al. (2010) desenvolveram um jogo para a aprendizagem de elicitação de requisitos de software, por meio do uso de situações e cenários simulados.

No que tange o acompanhamento do desempenho de alunos durante o processo de aprendizado em projeto de sistemas de software, a ferramenta SEREBRO fornece o desempenho de grupos no desenvolvimento de projetos de software, analisando a colaboração, a contribuição e o progresso de cada um [Hale, Jorgenson e Gamble 2011].

Práticas para apoio ao ensino de Engenharia de Software, sem o uso de ambientes computacionais, também tem sido criadas, como o jogo AprendES [Feitosa e Campos 2010]. Esse é um jogo baseado em cartas, que permite estimar o esforço para se construir um projeto, definindo-se o número de módulos do projeto utilizando-se cartas do tipo 'tamanho'. Já a ferramenta Modelando [Silva et al. 2012] é um jogo educacional para o ensino de Engenharia de Requisitos, que proporciona aos estudantes da disciplina um exercício prático, lúdico e iterativo com o objetivo de praticar os conceitos assimilados, utilizando-se os diagramas da UML (Linguagem de Modelagem Unificada).

Porém, nenhum dos ambientes de apoio ao aprendizado, citados anteriormente, envolve a análise dos requisitos para estimativa de esforço utilizando Planning Poker. Também não utilizam requisitos e esforços reais para apoio ao aprendiz de estimativa de software. Com base nos pontos fracos observados, na existência de poucos trabalhos na literatura neste contexto e os métodos mais comuns na indústria de software para realização de estimativas serem os baseados em julgamento de especialista [Jørgensen 2013b], foi desenvolvido um novo método e uma ferramenta para o ensino de estimativa de software, que podem ser aplicados em contextos reais de ensino, melhorando a qualidade na formação dos alunos nesta área.

\section{Metodologia e Ferramenta de Apoio ao Ensino Desenvolvida}

O objetivo dessa seção é apresentar a metodologia utilizada para o desenvolvimento e a análise de uma Ferramenta de Apoio ao Ensino de Estimativa de Software com Planning Poker, por meio de experimentos com alunos da UTFPR. Também é apresentada a ferramenta criada para testar o método desenvolvido. 
Primeiramente, foi desenvolvido o Fluxo de Execução dos Experimentos, conforme descrito nos diagramas das Figuras 1(a), 1(b), e 2. As Figuras 1(a), 1(b), e 2 correspondem a um único Diagrama de Atividades do experimento, que foi segmentado para uma melhor visualização, sendo, respectivamente, a primeira, a segunda e a terceira parte dos experimentos. Foram realizados quatro experimentos, cada um com duas etapas. Os experimentos 1 e 2 formaram a primeira iteração e os experimentos 3 e 4, a segunda iteração, conforme indicado na Tabela I. A Tabela I indica também as datas de realização de todos os experimentos.

Tabela I - Informações sobre a Condução dos Experimentos

\begin{tabular}{|c|c|c|c|c|c|c|c|c|}
\hline \multirow[b]{2}{*}{ Experimento } & \multicolumn{4}{|c|}{ Iteração 1} & \multicolumn{4}{|c|}{ Iteração 2} \\
\hline & \multicolumn{2}{|r|}{1} & \multicolumn{2}{|c|}{2} & \multicolumn{2}{|r|}{3} & \multicolumn{2}{|r|}{4} \\
\hline Curso & Engenharia de & $\begin{array}{l}\text { ia de } \\
\text { ção }\end{array}$ & \multicolumn{2}{|c|}{ Sistemas de } & \multicolumn{2}{|c|}{$\begin{array}{l}\text { Sistemas de } \\
\text { Informação }\end{array}$} & \multicolumn{2}{|c|}{$\begin{array}{l}\text { Engenharia de } \\
\text { Computação }\end{array}$} \\
\hline $\begin{array}{l}\text { Disciplina cursada pelos alunos } \\
\text { participantes }\end{array}$ & \multicolumn{2}{|c|}{$\begin{array}{l}\text { Análise e Projeto de } \\
\text { Sistemas }\end{array}$} & \multicolumn{2}{|c|}{$\begin{array}{l}\text { Engenharia de } \\
\text { Software } 2\end{array}$} & \multicolumn{2}{|c|}{$\begin{array}{l}\text { Análise e Projeto de } \\
\text { Sistemas }\end{array}$} & \multicolumn{2}{|c|}{$\begin{array}{l}\text { Análise e Projeto de } \\
\text { Sistemas }\end{array}$} \\
\hline Data da Introdução a Planning Poker & \multicolumn{2}{|c|}{$13 / 08 / 2014$} & \multicolumn{2}{|c|}{$18 / 08 / 2014$} & \multicolumn{2}{|c|}{$30 / 09 / 2014$} & \multicolumn{2}{|c|}{$29 / 10 / 2014$} \\
\hline Etapa & 1 & 2 & 1 & 2 & 1 & 2 & 1 & 2 \\
\hline Data da execução & $20 / 08 / 14$ & $27 / 08 / 14$ & $25 / 08 / 14$ & $25 / 08 / 14$ & $30 / 09 / 14$ & $07 / 10 / 14$ & $29 / 10 / 14$ & $26 / 11 / 14$ \\
\hline $\mathbf{N}^{\circ}$ de alunos na etapa & 14 & 12 & 28 & 28 & 15 & 13 & 20 & 22 \\
\hline
\end{tabular}

Na primeira etapa, os participantes tiveram uma introdução à prática do Planning Poker e, em grupos de três a quatro alunos, realizaram estimativas de esforço em homens/hora da implementação de 16 requisitos reais de um ambiente de desenvolvimento de software, oriundos de uma empresa de desenvolvimento de software comercial (ver as Figuras 1(a), 1(b), e 2). Na segunda etapa, os participantes foram apresentados ao mesmo conjunto de requisitos, porém, desta vez, tendo acesso a outras especificações de requisitos semelhantes, com os seus esforços reais, em homens/hora, apresentados. Esforços reais que também foram estimados utilizando-se Planning Poker, e realizados em ambiente de desenvolvimento da mesma empresa.

A ferramenta web faz a captura de diversos eventos do navegador, para acompanhar as ações dos alunos no processo de definição da estimativa, além dos dados inseridos pelos mesmos. Esta ferramenta foi construída utilizando-se: RubyOnRails 4.1.4, como framework de desenvolvimento back-end; jQuery 1.11.1, como framework de desenvolvimento front-end; MariaDB 5.5.39, como sistema gerenciador de banco de dados; Xubuntu 14.04 x64, como sistema operacional; Firefox 32, como navegador principal; Chrome 37, como navegador secundário.

$\mathrm{Na}$ primeira etapa do experimento, cada participante integrante do grupo de estimativa deveria se cadastrar, inserindo os seus dados pessoais e as suas competências profissionais e acadêmicas, conforme indicado pela atividade "Participantes cadastram os dados do participante" do diagrama da Figura 1(b). Também é solicitado o grau de conhecimento de competência, se básico, intermediário ou avançado.

Após o cadastro de todos os participantes, é possível selecionar, a partir de uma lista de requisitos, cada um dos requisitos e as suas especificações, a fim de ser feita a estimativa de esforço em horas para o mesmo. Esta descrição é relativa à atividade "Participantes leem os dados referentes ao requisito" do diagrama da Figura 2.

Ao preencherem os campos de valor de estimativa e de justificativa para cada estimador, exibidos na Figura 3, e clicarem em "Terminar rodada", os dados da rodada 
são gravados, e a tela será atualizada para permitir uma nova rodada, exibindo os valores anteriores estimados na coluna "Valor Anterior" (ver a Figura 3) para que o moderador possa saber se os estimadores estão chegando a um consenso. Esta ação compreende a atividade "Participantes realizam as rodadas de jogo, definindo e justificando o esforço estimado" do diagrama da Figura 2.

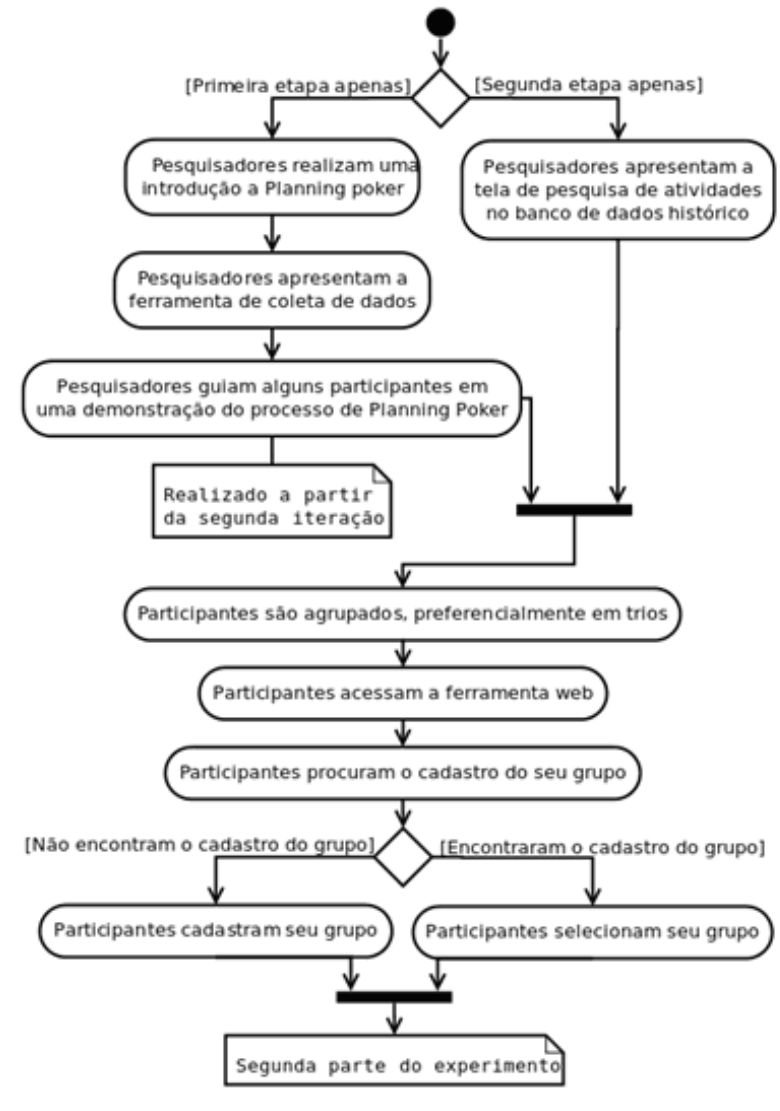

(a)

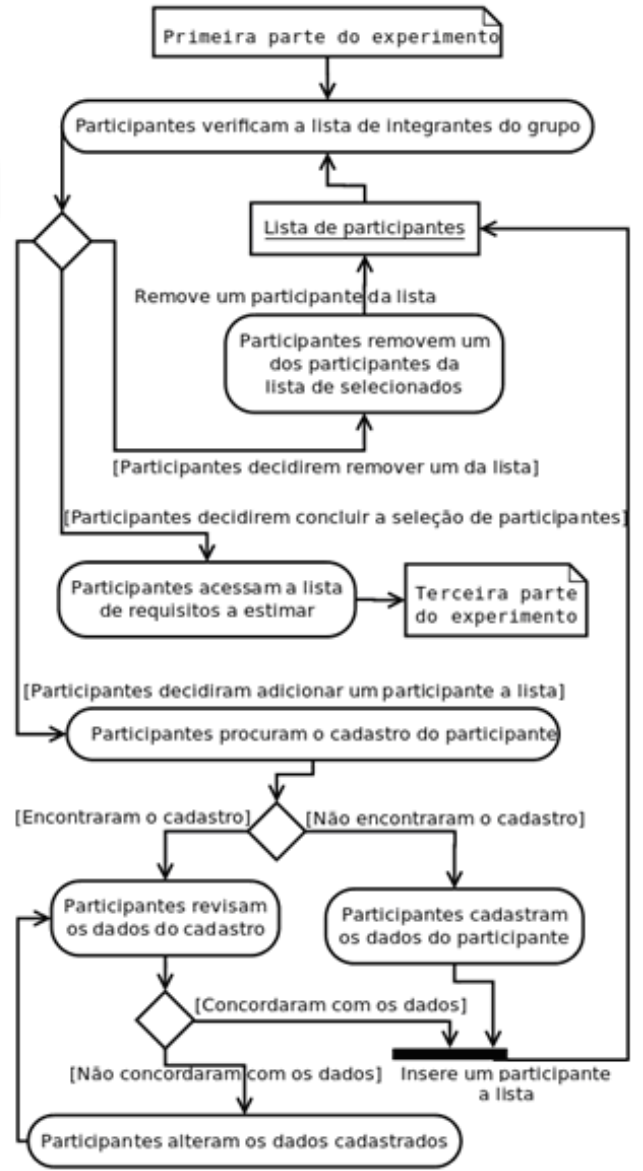

(b)

Figura 1 - (a) Diagrama do Fluxo de Execução do Experimento - Primeira Parte. (b) Diagrama do Fluxo de Execução do Experimento - Segunda Parte.

Caso os participantes cheguem a um consenso ou o moderador decida encerrar as rodadas por falta de consenso, os participantes devem definir o valor da estimativa final e clicar em "Definir estimativa final" (ver a Figura 3), o que leva a uma nova tela. Nessa nova tela os participantes devem, então, preencher os campos de estimativa final e de razões e incertezas. Esta ação compreende a atividade "Participantes definem a estimativa final e a justificam" do diagrama da Figura 2. As razões e incertezas podem estar relacionadas a fatores como complexidade, dificuldade da linguagem de programação, profundidade insuficiente dos requisitos, entre outros.

Apenas na segunda etapa, a fim de verificar o impacto da utilização da busca em repositório histórico durante a execução de estimativas utilizando Planning Poker, os alunos puderam fazer buscas por palavras chaves relativas aos requisitos a serem estimados, por meio da opção "Participantes decidiram buscar estimativas no banco de dados, apresentada na Figura 2. 
Ao preencherem os filtros de palavras por projeto e/ou por esforço executado mínimo e máximo, são exibidas as atividades de desenvolvimento de software encontradas. Esta ação compreende a atividade "Participantes pesquisam estimativas no banco de dados" do diagrama apresentado na Figura 2.

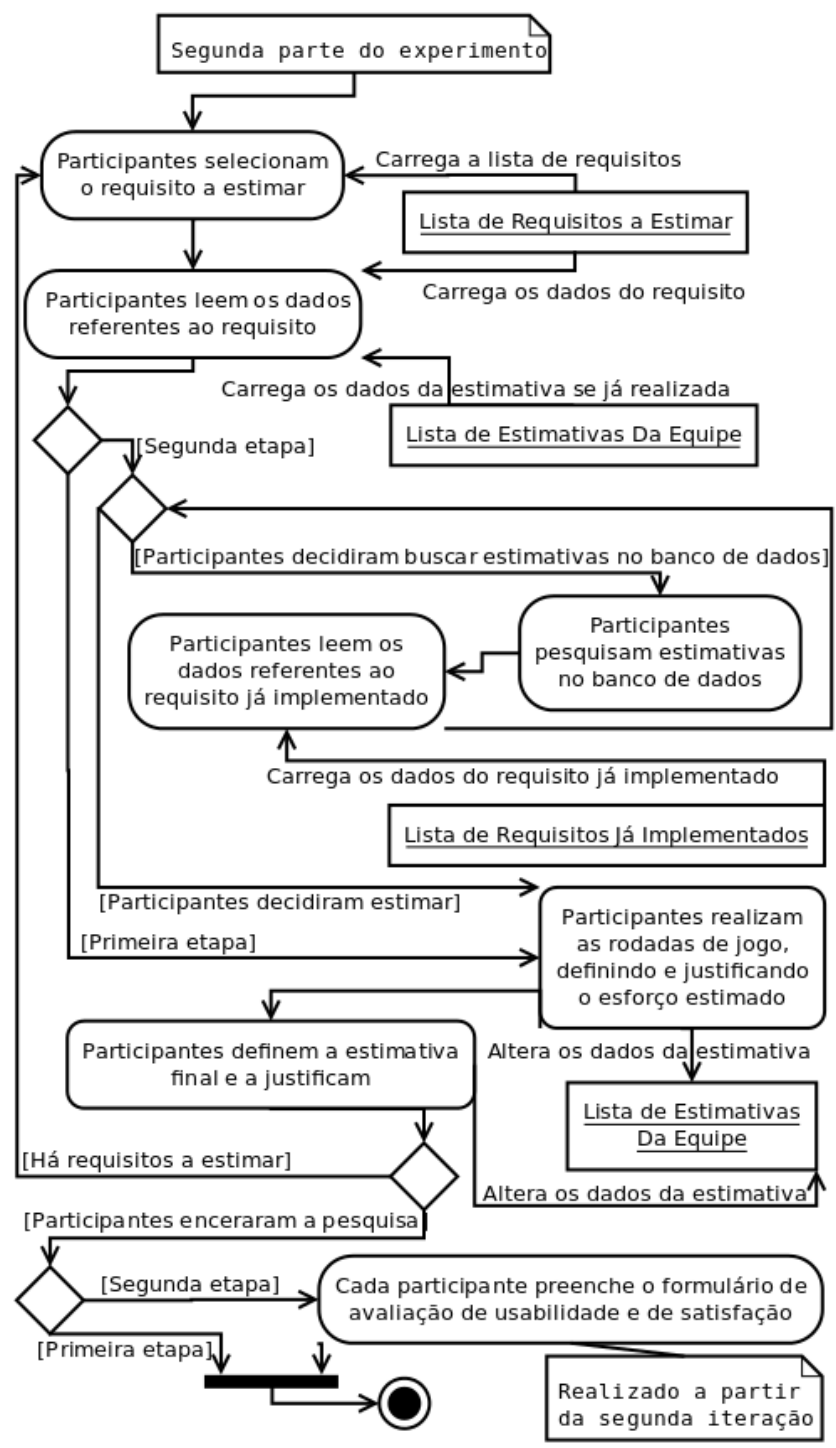

Figura 2 - Diagrama do Fluxo de Execução do Experimento - Terceira Parte.

Ao escolher uma atividade, os dados desta são carregados para a tela, com uma configuração de informações similar a configuração das telas de estimativa (ver a Figura 3), porém com as informações de "esforço realizado" relativo à implementação da atividade. Esta ação compreende a atividade "Participantes lêem os dados referentes ao requisito já implementado" do diagrama da Figura 2. Desta forma, é possível aos participantes buscar requisitos semelhantes ao requisito que está sendo analisado, a fim de fazer uma estimativa a partir de valores reais de tempo gasto para implementar requisitos semelhantes.

Para avaliar a ferramenta, um questionário foi desenvolvido, revisado e aplicado ao final do terceiro e do quarto experimentos. O questionário tem respostas fechadas, sendo apresentado, com os resultados, na Seção 5: Resultados, Análise e Discussão. 
CBIE-LACLO 2015

Anais do XXVI Simpósio Brasileiro de Informática na Educação (SBIE 2015)
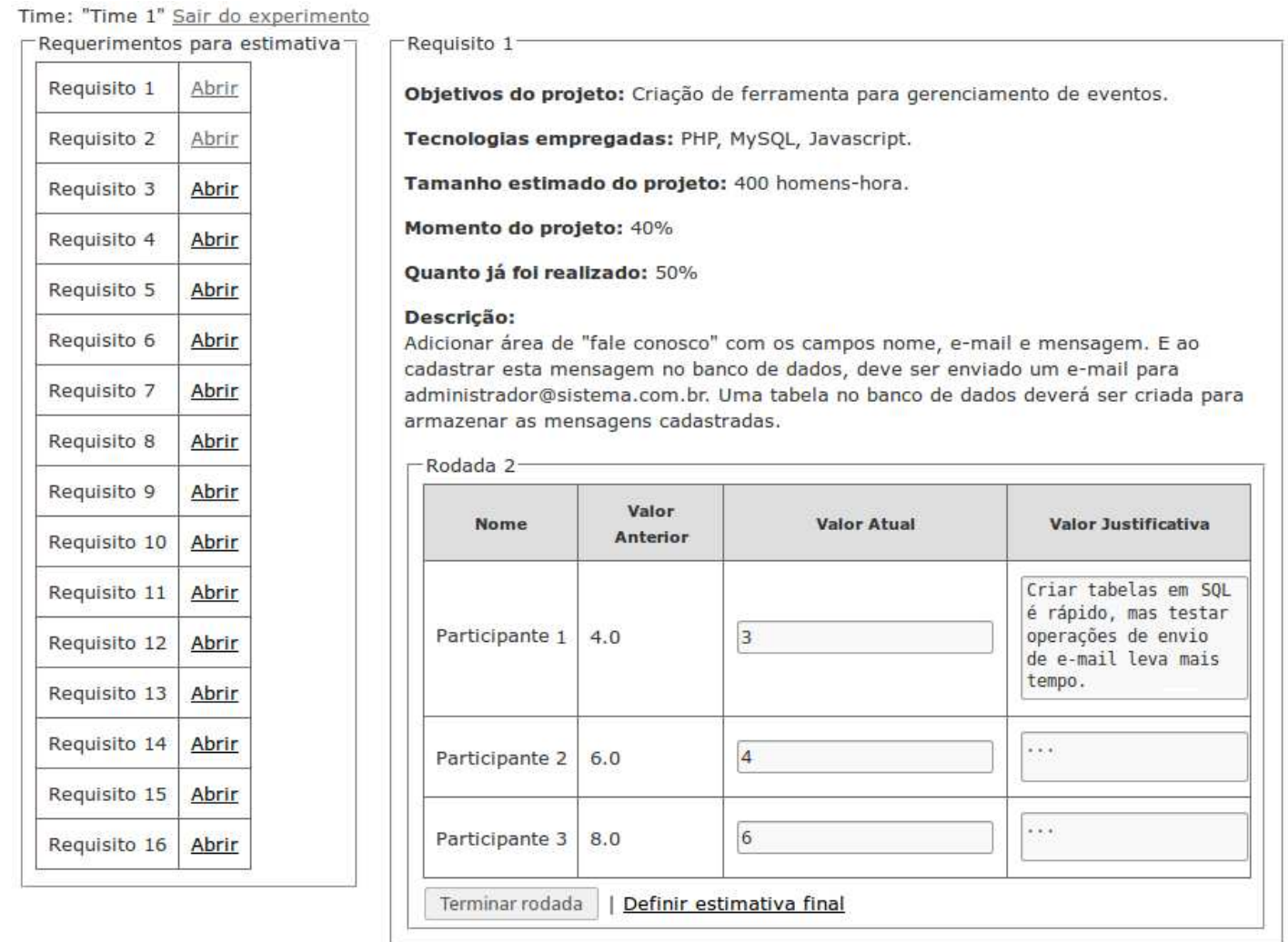

Figura 3 - Tela de Exibição do Requisito a Estimar e de Execução das Rodadas de Planning Poker.

\section{Resultados, Análise e Discussão}

No total, participaram 79 alunos, divididos em 24 grupos, dos quais foram desconsiderados grupos que: tiveram falta de seus participantes na segunda etapa do experimento, incluíram componentes no grupo na segunda etapa do experimento e analisaram menos da metade do total dos 16 requisitos analisados. O resultado da comparação pode ser visto na Tabela II.

Após a definição dos grupos que seriam considerados, foi aplicada a fórmula da Média das Diferenças Absolutas (MDA), indicada pela equação (1):

$$
M D A=\frac{\sum_{i=1}^{n}\left|x_{i}-\hat{x}_{i}\right|}{n}
$$

A equação (1), onde " $i$ " representa o número da estimativa, " $n$ " representa o número total de estimativas, " $x_{i}$ " representa o esforço real, $\mathrm{e}$ " $\hat{x}_{i}$ " representa o esforço estimado, foi aplicada para cada estimativa de cada requisito na primeira e na segunda etapas, para cada um dos grupos (ver a Tabela II).

Uma comparação da melhoria de acurácia das estimativas dos grupos em seus experimentos pode ser vista na Tabela II. Da Tabela II verifica-se que, dos 14 grupos analisados, 11 tiveram uma melhora da acurácia das estimativas e 3 tiveram uma piora 
da acurácia das estimativas entre as etapas 1 e 2 , representadas na Tabela II como a melhoria relativa da média das diferenças absolutas (MRMDA).

Tabela II - Comparação da Acurácia dos Grupos entre as etapas 1 e 2

\begin{tabular}{|c|c|c|c|c|c|}
\hline Iteração & Experimento & Grupo & MDA $_{\mathbf{1}}$ (Etapa 1) & MDA $_{\mathbf{2}}$ (Etapa 2) & Melhoria das estimativas entre as etapas 1 e 2(MRMDA) \\
\hline 1 & 2 & 6 & 3,46 & 3,4 & $1,63 \%$ \\
\hline 1 & 2 & 7 & 1,51 & 3,67 & $-143,73 \%$ \\
\hline 1 & 2 & 8 & 3,26 & 2,12 & $34,86 \%$ \\
\hline 1 & 2 & 9 & 11,06 & 3,28 & $70,31 \%$ \\
\hline 1 & 2 & 10 & 2,98 & 1,83 & $38,66 \%$ \\
\hline 1 & 2 & 11 & 6,05 & 2,03 & $66,5 \%$ \\
\hline 1 & 2 & 12 & 7,93 & 6,83 & $13,88 \%$ \\
\hline 1 & 2 & 13 & 0,82 & 1,0 & $-20,92 \%$ \\
\hline 1 & 2 & 14 & 2,67 & 1,43 & $46,37 \%$ \\
\hline 2 & 3 & 16 & 1,15 & 0,48 & $58,23 \%$ \\
\hline 2 & 3 & 18 & 1,95 & 0,99 & $49,28 \%$ \\
\hline 2 & 4 & 19 & 2,35 & 0,66 & $72,17 \%$ \\
\hline 2 & 4 & 20 & 2,68 & 3,77 & $-40,75 \%$ \\
\hline 2 & 4 & 23 & 2,06 & 0,72 & $64,84 \%$ \\
\hline
\end{tabular}

Na Tabela II, quando há melhoria nas estimativas, ou seja, a diferença entre o valor real o estimado diminui entre etapas, MRMDA é positivo, sendo negativo quando houve aumento na diferença. Os valores para MRMDA resultam da equação (2):

$$
\operatorname{MRMDA}\left(M D A_{1}, M D A_{2}\right)=\left\{\begin{array}{ccc}
s e M D A_{1}>M D A_{2} & \frac{\min \left(M D A_{1}, M D A_{2}\right)}{\max \left(M D A_{1}, M D A_{2}\right)} & -1 \\
\text { señ̃o } & \frac{\max \left(M D A_{1}, M D A_{2}\right)}{\min \left(M D A_{1}, M D A_{2}\right)} & -1
\end{array}\right\} \times-100
$$

A correlação de Pearson entre a média das notas de cada grupo relativas às disciplinas da área de Computação que os alunos cursaram e a MRMDA foi $r=-0,26$. Na Figura 4, é apresentado o gráfico de dispersão relativo a essa correlação.

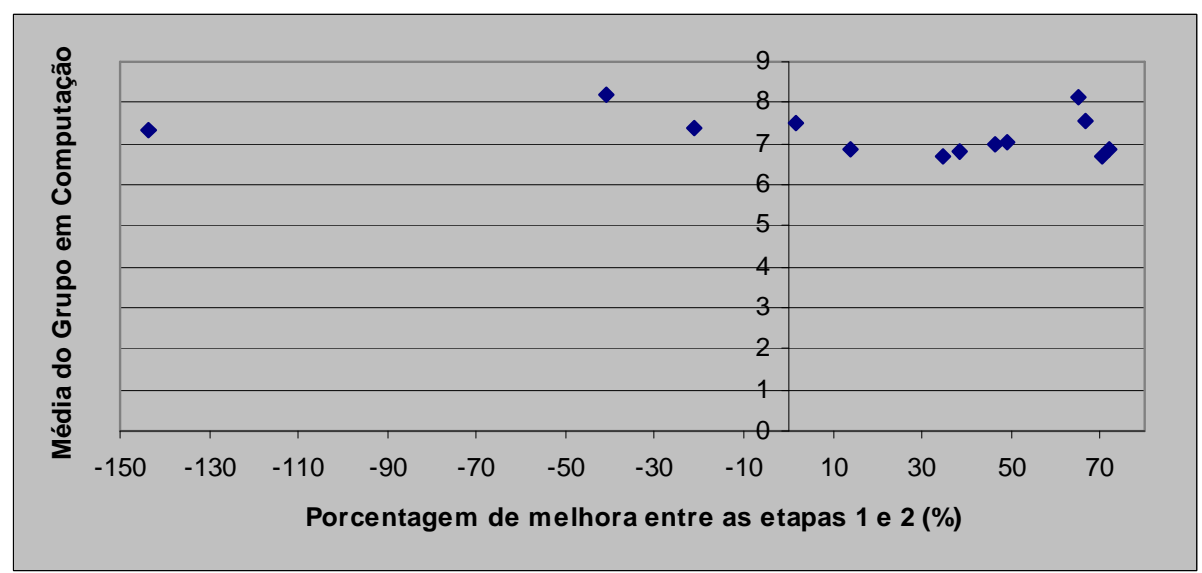

Figura 4 - Gráfico de dispersão entre a média das notas de cada grupo e a MRMDA.

O questionário desenvolvido, e aplicado para avaliar a ferramenta, está apresentado com os seus resultados na Tabela III, sendo: CPle, Concordo plenamente; CPar, Concordo parcialmente; Ind, Indiferente; DPar, Discordo parcialmente; DCom, Discordo completamente. 
Tabela III - Questionário de Avaliação da Ferramenta.

\begin{tabular}{|l|c|c|c|c|c|}
\hline \multicolumn{1}{|c|}{ Questões } & CPle & CPar & Ind & DPar & DCom \\
\hline 1. A ferramenta web tem tema bem definido e este é mantido em todas as páginas. & $83,3 \%$ & $16,7 \%$ & $0,0 \%$ & $0,0 \%$ & $0,0 \%$ \\
\hline 2. O objetivo da ferramenta foi alcançado. & $70,0 \%$ & $30,0 \%$ & $0,0 \%$ & $0,0 \%$ & $0,0 \%$ \\
\hline 3. O conteúdo da ferramenta é interessante para seu público-alvo. & $83,3 \%$ & $16,7 \%$ & $0,0 \%$ & $0,0 \%$ & $0,0 \%$ \\
\hline 4. O usuário descobre facilmente onde clicar para chegar à página que deseja. & $40,0 \%$ & $43,3 \%$ & $6,7 \%$ & $10,0 \%$ & $0,0 \%$ \\
\hline 5. A ferramenta web funcionou corretamente com o(s) navegador(es) utilizado(s). & $93,3 \%$ & $3,3 \%$ & $3,3 \%$ & $0,0 \%$ & $0,0 \%$ \\
\hline 6. A ferramenta web tem um leiaute que auxilia a localização de todos os elementos. & $13,3 \%$ & $36,7 \%$ & $13,3 \%$ & $36,7 \%$ & $0,0 \%$ \\
\hline 7. Utilizar a ferramenta me auxiliou a compreender o processo de estimativa. & $66,7 \%$ & $26,7 \%$ & $6,7 \%$ & $0,0 \%$ & $0,0 \%$ \\
\hline 8. Utilizar a ferramenta me auxiliou a compreender o Planning Poker. & $90,0 \%$ & $6,7 \%$ & $3,3 \%$ & $0,0 \%$ & $0,0 \%$ \\
\hline 9. Tenho interesse em utilizar a ferramenta para melhorar minhas estimativas. & $20,0 \%$ & $50,0 \%$ & $30,0 \%$ & $0,0 \%$ & $0,0 \%$ \\
\hline 10. Tenho interesse em utilizar a ferramenta para realizar estimativas de forma prática. & $23,3 \%$ & $43,3 \%$ & $33,3 \%$ & $0,0 \%$ & $0,0 \%$ \\
\hline
\end{tabular}

\section{Conclusões}

Neste artigo é apresentado o método denominado Estimativa de Software com Revisão de Atividades Executadas Anteriormente, utilizando Planning Poker. Tal método foi testado com alunos de graduação por meio de sua implementação em uma ferramenta web, que reforça a correta aplicação do Planning Poker, permite o armazenamento de dados gerados na discussão, e facilita a revisão de estimativas antigas em base histórica.

Dos 24 grupos que participaram dos experimentos, foi possível manter 14 grupos para a análise dos resultados, em virtude das faltas ou entradas de alunos nos grupos. Isso deve ser levado em conta por pesquisadores que queiram realizar experimentos com alunos de graduação em um meio acadêmico.

O método de Revisão de Atividades Executadas, utilizando Planning Poker, melhorou a estimativa de esforço em $78,6 \%$ dos grupos analisados, podendo ser um importante método para aprimorar o processo de desenvolvimento de software e para treinamento de alunos e profissionais na área de estimativa de software. A ferramenta desenvolvida para aplicar e testar o método auxiliou a grande maioria dos alunos a compreender o processo de estimativa de software e o Planning Poker.

Praticamente não houve correlação entre a média das notas de cada grupo, relativas a disciplinas da área de Computação que os alunos cursaram, e a melhoria relativa da média das diferenças absolutas (MRMDA) $(r=-0,26)$, o que pode ser confirmado ao analisar o gráfico de dispersão (Figura 4). Ou seja, o grau de aproveitamento em disciplinas anteriormente cursadas na área de Computação não influenciou na melhoria das estimativas.

\section{Referências}

Cohn, M. (2005) “Agile estimating and planning”. Prentice Hall Professional Technical Reference.

Dagnino, A. (2013) "Estimating software-intensive projects in the absence of historical data". In Software Engineering (ICSE), 2013 35th International Conference on, pages 941-950.

Desharnais, J.-M., Buglione, L., and Kocatürk, B. (2011) "Using the cosmic method to estimate agile user stories". In Proceedings of the 12th International Conference on Product Focused Software Development and Process Improvement, Profes '11, pages 68-73, New York, NY, USA. ACM. 
Feitosa, A. C., Campos, G. M. M. (2010) “AprendES: um jogo educacional para auxiliar o processo de ensino-aprendizagem da Engenharia de Software”. In: XXI Simpósio Brasileiro de Informática na Educação, João Pessoa, Brasil, pp. 1-4.

Grenning, J. (2002) "Planning poker or how to avoid analysis paralysis while release planning". White Paper, Renaissance Software Computing,

Gruschke, T. M. and Jørgensen, M. (2008) "The role of outcome feedback in improving the uncertainty assessment of software development effort estimates". ACM Trans. Softw. Eng. Methodol., 17(4):20:1-20:35.

Hale, M., Jorgenson, N., Gamble, R. (2011) "Predicting Individual Performance in Student Project Teams". In: 24th IEEE Conference on Software Engineering Education and Training, Honolulu, Estados Unidos, pp.11-20.

Jørgensen, M. (2013) "Relative estimation of software development effort: It matters with what and how you compare". IEEE Software, 30(2):74-79.

Jørgensen, M. and Grimstad, S. (2008) "Avoiding irrelevant and misleading information when estimating development effort". IEEE Softw., 25(3):78-83.

Jørgensen, M. and Gruschke, T. M. (2009) "The impact of lessons-learned sessions on effort estimation and uncertainty assessments". IEEE Trans. Softw. Eng., 35(3):368383.

Mahnic, V. and Hovelja, T. (2011) "On using planning poker for estimating user stories". Journal of Systems and Software, 85(9):2086 - 2095. Selected papers from 2011 Joint Working IEEE/IFIP Conference on Software Architecture (WICSA 2011).

Raith, F., Richter, I., Lindermeier, R., and Klinker, G. (2013) "Identification of inaccurate effort estimates in agile software development". In Software Engineering Conference (APSEC, 2013 20th Asia-Pacific, volume 2, pages 67-72.

Santos R., Lima T., Campos B., Werner C. (2013) "Experiência na Avaliação de Efetividade de um Jogo para Ensino de Conceitos de Requisitos em Sistemas Ubíquos”. In: XXIV Simpósio Brasileiro de Informática na Educação, Campinas, Brasil, pp. 935-939.

Silva, J. C., Sousa, S. P. A., Kulesza, R., Brito, A. V. (2012) "Uma avaliação do emprego do jogo Modelando como apoio ao ensino de Engenharia de Requisitos". In: XX Workshop sobre Educação em Computação, Curitiba, Brasil, 1-11.

Thiry M., Zoucas A., Gonçalves R. Q. (2010) "Promovendo a Aprendizagem de Engenharia de Requisitos de Software Através de um Jogo Educativo". In: XXI Simpósio Brasileiro de Informática na Educação, João Pessoa, Brasil, pp. 1-10.

Vargas, D., Moro, T., Dambrósio, G., Cassal, M., Bernardi, G., Cordenonsi, A. Z (2010) "Desenvolvimento de um Jogo de Empresa baseado em Agentes de Software e Instituições Eletrônicas para simulação de Elicitação de Requisitos de Software". In: XVIII Workshop sobre Educação em Informática, Belo Horizonte, Brasil, 847-856.

Zuppiroli, S., Ciancarini, P., Gabbrielli, M. (2012) "A Role-Playing Game for a Software Engineering Lab: Developing a Product Line". In: 25th IEEE Conference on Software Engineering Education and Training, Nanjing, China, pp.13-22. 